\title{
Rare case of upside-down stomach in advanced hiatal hernia
}

\author{
Nooraldin Merza MD, John Lung BS, Omar Bazzaz MD, Farah Aljuboory MD, Mazin Saadaldin MD, FRCS
}

\begin{abstract}
Upside-down stomach (UDS) is the rarest type of hiatal hernia; it involves migration of the entire stomach into the posterior mediastinum with volvulus. We report a patient with chronic gastroesophageal reflux disease (GERD) and myelodysplastic syndrome who was admitted for syncope and signs of aspiration pneumonia with an incidental findings of hiatal hernia type IV with UDS. The patient had been experiencing generalized weakness and had a history of multiple falls without head injury. He was not in acute distress. The chest $x$-ray was suspicious for pneumonia. The patient had a hemoglobin level of $6.8 \mathrm{~g} / \mathrm{dl}$ and was transfused with multiple units of packed erythrocytes during his hospitalization for treatment of his anemia secondary to myelodysplastic syndrome. Computed tomography of the thorax revealed a large hiatal hernia type IV with UDS in the posterior mediastinum. Surgical intervention was recommended, but the patient elected to postpone surgery due to the lack of symptoms. He died one month later from complications related to myelodysplastic syndrome. The diagnosis of upsidedown stomach can be suspected on chest $x$-ray but requires a high degree of suspicion. All surgical interventions for UDS must consider the overall prognosis of the patient and estimated improved quality of life before repair, especially given the high recurrence rate of UDS after surgical repair.
\end{abstract}

Keywords: Upside-down stomach, hiatal hernia, paraesophageal hernia, gastric incarceration, gastric outlet obstruction, gastric volvulus

\section{INTRODUCTION}

An upside-down stomach (UDS) is the rarest type of hiatal hernia ( $<5 \%$ of all hiatal hernias) and is defined by the migration of the entire stomach into the posterior mediastinum. ${ }^{1,2}$ These patients can present with a spectrum of findings and symptoms ranging from reflux disease to mechanically impaired gastric emptying; they can develop gastric volvulus. The upside-down stomach is associated with a risk of incarceration and volvulus development which can

Corresponding author: Nooraldin Merza Contact Information: Nooraldin.Merza@ttuhsc.edu DOI: 10.12746/swrccc.v7i31.581 be complicated by acute gastric outlet obstruction, advanced ischemia, gastric bleeding, and perforation. ${ }^{1}$ We report a patient with chronic gastroesophageal reflux disease (GERD) and myelodysplastic syndrome who was admitted for syncope and signs of aspiration pneumonia with an incidental finding of hiatal hernia type IV with UDS.

\section{CASE}

A 74-year-old Caucasian man with GERD and myelodysplastic syndrome diagnosed three months prior to admission, chronic asymptomatic hiatal hernia diagnosed two years ago, two episodes of diverticulitis two years ago, rheumatoid arthritis, osteoporosis, hypothyroidism, hyperlipidemia, and chronic anemia presented to the emergency department with 
syncope. The patient had been experiencing generalized weakness and had a history of multiple falls without head injury. He had a one-week history of chills without fever and a productive cough with clear sputum. He had loss of appetite for the past six months with dysgeusia and a 30-pound weight loss over the last 10 months. He had received packed red blood cells (RBCs) and darbepoetin alfa monthly since his diagnosis and recently was transfused two units one month prior to presentation.

On admission, the patient had a temperature of $37.8^{\circ} \mathrm{C}$ and a peripheral oxygen saturation of $91 \%$. Other vital signs were within normal limits. The patient was not in acute distress, and his chest examination was clear to auscultation bilaterally without diminished air entry or crepitation or other sounds. Cardiovascular system examination was normal. His abdomen was soft, nontender, and nondistended without hepatosplenomegaly.

The chest $x$-ray suggested pneumonia, and a five-day course of oral levofloxacin was started. His hemoglobin level was $6.8 \mathrm{~g} / \mathrm{dl}$, RBC $2.55 \times 10^{6} / \mu \mathrm{L}$, and hematocrit $22 \%$. He was transfused with multiple units of packed RBCs during his hospitalization for treatment of his anemia secondary to myelodysplastic syndrome. Computed tomography of the thorax (CT) revealed a large hiatal hernia type IV with UDS in the posterior mediastinum (Figure 1). A CT abdomen/pelvis from seven months prior showed the entire stomach within the chest. An x-ray upper gastrointestinal series with diatrizoic acid showed significant narrowing of the gastroesophageal junction and delayed gastric emptying (Figure 2). The patient had tracheal aspiration during swallow evaluation. Surgical intervention was recommended, but the patient elected to postpone surgery and was discharged. Hospice care was initiated two weeks later due to his worsening myelodysplastic syndrome, and he died one month later.

\section{Discussion}

The major cause of hiatal hernias is the loosening of the diaphragmatic crura through aging or congenital defects. According to the anatomical abnormality,

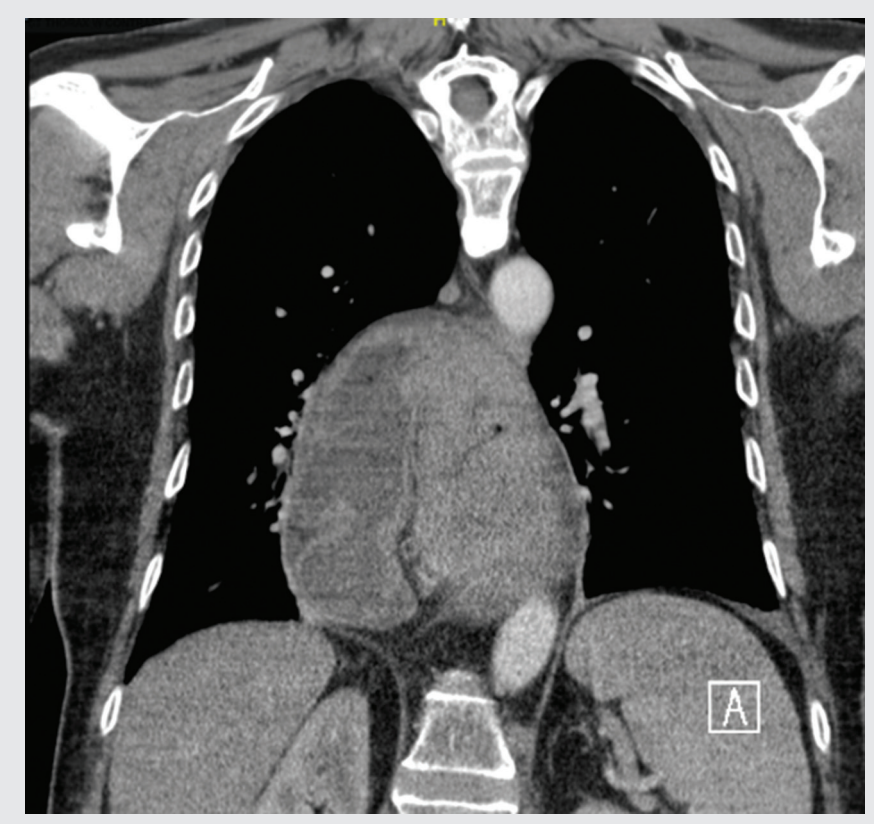

Figure 1. CT-Thorax without contrast showing a large retrocardiac hiatal hernia with entire stomach in the upside-down position.

it can categorized by the shape: type I (sliding hernia, the most common), type II hernia (paraesophageal hernia, characterized by paraesophageal herniation of the gastric fundus but with the gastroesophageal junction in a normal abdominal position), type III hernia (mixed hernia, both gastroesophageal junction and the gastric fundus in the posterior mediastinum), and type IV hernia (rare and defined by intrathoracic stomach). ${ }^{3}$ Since both the gastroesophageal junction and parts of the stomach migrate into the thorax, UDS represents a large mixed type-sliding and paraesophageal (type 3) hernia. ${ }^{2,4}$ However, many authors refer to UDS as a type 4 hiatal hernia. ${ }^{5}$ Our patient was diagnosed with a large asymptomatic sliding type I hiatal hernia measuring $10 \mathrm{~cm} \times 11 \mathrm{~cm} \times 7 \mathrm{~cm}$ two years prior to presentation; this hernia had never caused him symptoms.

The clinical significance of an UDS involves the risk of incarceration and strangulation. In stable patients with acute presentation of large paraesophageal hernia or UDS exhibiting acute mechanical 


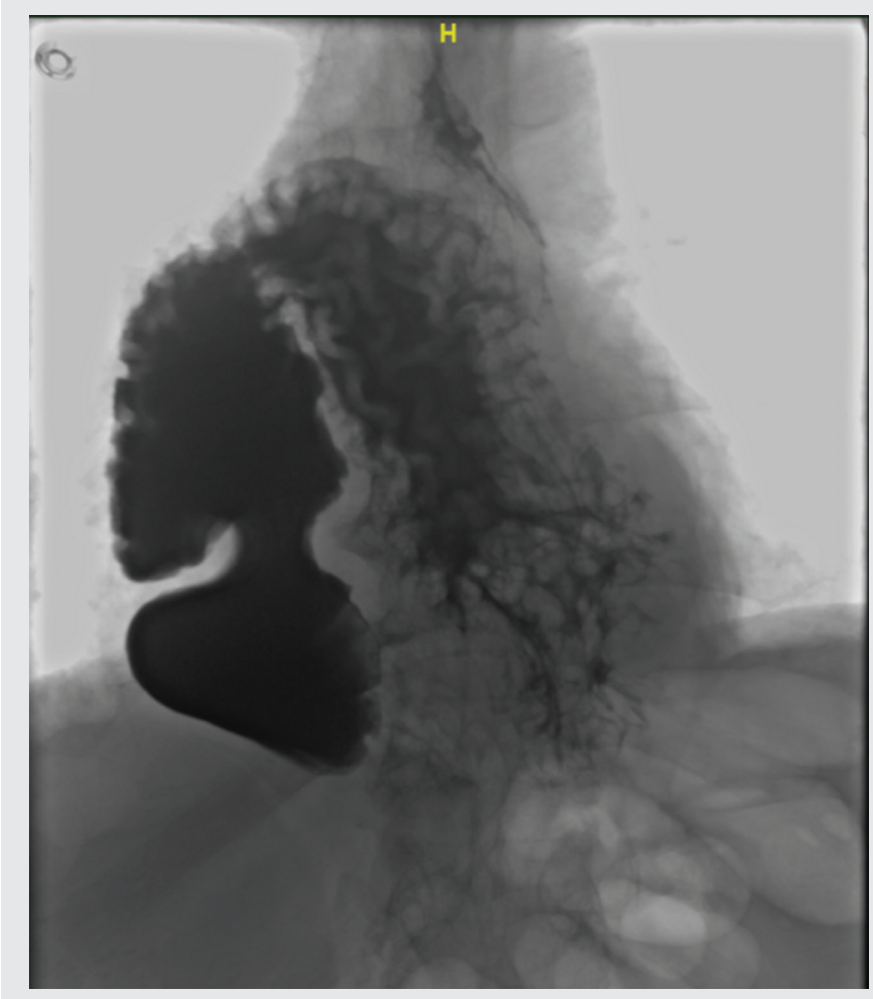

Figure 2. Upper GI x-ray with diatrizoic acid showing a large retrocardiac hiatal hernia with entire stomach in the upside-down position, causing significant narrowing at the gastroesophageal junction and delayed gastric emptying.

gastric outlet obstruction, laparoscopic reduction and hernia repair followed by an anti-reflux procedure are needed. These patients can develop life-threatening complications, such as ischemia, gastric perforation, or severe bleeding, with an incarcerated UDS and require emergent surgery. ${ }^{1}$ Due to high rates of mortality (up to $17 \%$ ) with emergency hiatal hernia repair surgery, elective repair is indicated for UDS even in asymptomatic patients. ${ }^{6}$ Options for surgery include laparoscopic repair, which is associated with high success rates, lower postoperative morbidity, and shorter hospital stays compared to conventional laparotomy or thoracotomy. Sutured crural repair was common before the laparoscopic repair became available. Both are associated with high rates of recurrence
(8-27\% in laparoscopic repair, $42 \%$ and higher for crural repair).

The diagnosis of upside-down stomach can be suspected on chest $\mathrm{x}$-ray but requires a high degree of suspicion. The diagnosis is difficult and is usually based on gastrografin or barium studies. ${ }^{7}$ In our case, the patient was asymptomatic and the upsidedown stomach was found incidentally on a CT scan of the thorax to evaluate him for recurrent pneumonia. Because of worsening complications related to myelodysplastic syndrome, the patient opted for no intervention and a possible clinical trial for myelodysplastic syndrome in a larger city. However, the patient soon choose palliative care due to travel distances and died a week later. A surgical intervention would not have improved the patient's quality of life given the patient's overall poor prognosis and lack of symptoms related to his type IV UDS hiatal hernia.

\section{Conclusion}

All surgical interventions for UDS must consider the overall prognosis of the patient and estimated improved quality of life before repair, especially given the high recurrence rate of UDS even after surgical repair.

Article citation: Merza N, Lung J, Bazzaz O, Aljuboory F, Saadaldin M. Rare case of upside-down stomach in advanced hiatal hernia. The Southwest Respiratory and Critical Care Chronicles 2019;7(31):52-55

From: Department of Internal Medicine (NM, MS) and School of Medicine (JL) Texas Tech University Health Sciences Center, Amarillo, Texas; Department of Internal Medicine (OB), Veterans Affairs Medical Center, Amarillo, Texas; BSA Health System (FA), Amarillo, Texas

Submitted: 9/22/2019

Accepted: 10/7/2019

Reviewer: Kenneth Nugent MD

Conflicts of interest: none

This work is licensed under a Creative Commons Attribution-ShareAlike 4.0 International License. 


\section{REFERENCES}

1. Schiergens TS, Thomas MN, Hüttl TP, et al. Management of acute upside-down stomach. BMC Surg 2013;13:55.

2. Hill, LD, Tobias JA. Paraesophageal hernia. Arch Surg 1968; 96(5),735-744.

3. Krähenbühl L, Schäfer M, Farhadi J, et al. Laparoscopic treatment of large paraesophageal hernia with totally intrathoracic stomach. JACS 1998;187(3),231-237.

4. Wo JM, Branum GD, Hunter JG, et al. Clinical features of type III (mixed) paraesophageal hernia. Am J Gastroenterol 1996;91(5),914-916.
5. Obeidat FW, Lang RA, Knauf A, et al. Laparoscopic anterior hemifundoplication and hiatoplasty for the treatment of upside-down stomach: mid- and long-term results after 40 patients. Surg Endosc 2011;25(7):2230-2235.

6. Sakran N, Nevo H, Dar R, et al. Laparoscopic repair of a large paraesophageal hernia with migration of the stomach into the mediastinum creating an upside-down stomach. Case Rep Surg 2017;2017:7428195.

7. Shivanand G, Seema S, Srivastava DN, et al. Gastric volvulus. Clinical Imaging 2003;27(4),265-268. 